\title{
Analysing change over time for small areas - guidance
}

\author{
By The Area Based Analysis Unit, Office for National Statistics ${ }^{1}$
}

\section{Abstract}

A strategy for analysing change over time for small areas has been developed and published by ONS. This article provides an introduction to the strategy and illustrates the principles with a simple case study using out-of-work benefit data as a proxy for 'worklessness'. It aims to show that, although comparison of change over time has some complexities, it is manageable when broken down into stages.

The 5 stage strategy is supported by a free interactive tool that also focuses on benefit data to illustrate the strategy and automates the creation of box plots, maps and histograms for every local authority in the country. This article is intended to give advice to those carrying out their own analysis and should be of interest to anyone wanting to develop a good understanding of how things are changing in their own area.

It will help you produce a valid analysis that could form the basis for targeting resources or checking whether existing policy interventions are having the desired effect - helping to answer questions such as, 'Has my area improved since last year?' or 'Is the gap between our disadvantaged and non-disadvantaged areas reducing?'

\section{Introduction}

Understanding change in conditions is a critical part of developing and monitoring policies for small areas. A good understanding of how things have changed can play a critical role in targeting resources or in checking whether existing policy interventions are having the desired effect.

A range of small area data supporting the current interest in neighbourhood renewal, regeneration and improved local intelligence is available through websites such as Neighbourhood Statistics. However, the process of analysing and understanding change in these data brings a range of issues and can be complex and daunting.

ONS has recently developed and published a strategy for analysing change over time for small areas. This article provides a summary of the approach recommended and should be of interest to anyone trying to answer questions such as:

- How has my local authority changed compared with others in the region?

- Is the gap closing between the most disadvantaged areas and the rest?

- Which small areas have experienced significant change?

The strategy does not recommend a 'best' method, but instead provides options for analysts to consider, and advice on how to avoid the pitfalls of studying change.

1 The main contributors on this article were Nicola White, Angela Medland, Steve Bond and Alistair Calder.

Note: This article is necessarily a brief summary of the approach and issues identified. The full guidance document 'Analysing Change over Time for Small Areas - A Strategy' provides a full discussion of the steps outlined here and the issues to consider during analysis. In particular the strategy document provides a full discussion of the importance of data quality and the key issues relating to data - variability, spatial diversity, volatility, and independence which are not covered here. If you want to carry out your own analysis, please make use of the full guidance document, which is available to download from the Analysis and Guidance section of the Neighbourhood Statistics website: www.neighbourhood.statistics.gov.uk or, on request, from better.info@ons.gsi.gov.uk. This article assumes a basic knowledge of statistical methods and measures. The full guidance document and the guidance area of the Neighbourhood Statistics website provide further advice on the terms used. 
The 5 stage strategy

The approach outlined has 5 stages:

1) Selecting the data

2) Understanding the context of your analysis

3) Splitting the data into groups for analysis

4) Understanding how the groups are changing

5) Testing the significance of individual changes

This article provides an introduction to each of these stages and illustrates the principles using a simple case study. In particular, the article focuses on elements of stages 4 and 5 looking at whether gaps are changing between groups and testing the significance of individual changes. The approach has been developed with help from 12 local authorities across the country and it is hoped that the approaches outlined here will be of interest and applicable to other local analyses.

We illustrate the proposed strategy using out-of-work benefit data, as a proxy for 'worklessness' and with a case study area created to mimic a local authority. Although the data are selected for a fictitious area they are based on published benefit data taken from within a real region.

Figure 7.1 Flowchart: Steps used in the 5 stages of analysis

\begin{tabular}{|c|}
\hline $\begin{array}{l}\text { 1. Select your data } \\
\text { - Select a topic of interest } \\
\text { - Select a geographic area } \\
\text { - Select a timeframe } \\
\text { - Decide whether to use count or rate data (or both) }\end{array}$ \\
\hline$\downarrow$ \\
\hline $\begin{array}{l}\text { 2. Understand the context of your analysis } \\
\text { - How does your local authority (LA) compare with the region? } \\
\text { - How does your area compare with other LAs? } \\
\text { - How do changes in your LA compare with other LAs in the } \\
\text { region? } \\
\text { - How is the data distributed within the LA? } \\
\text { - Has the overall spread of the data changed? } \\
\text { - Are there any obvious spatial patterns? } \\
\text { - How has each Lower Layer Super Output Area (LSOA) in your } \\
\text { LA changed from time } 1 \text { to time } 2 \text { ? }\end{array}$ \\
\hline$\downarrow$ \\
\hline $\begin{array}{l}\text { 3. Split your area into groups for analysis } \\
\text { - Determine how you want to split your area } \\
\text { - Split your data into groups }\end{array}$ \\
\hline$\downarrow$ \\
\hline $\begin{array}{l}\text { 4. Understand how the groups are changing } \\
\text { - Has the spread in the upper half of the data distribution } \\
\text { changed? } \\
\text { - How are the groups increasing or decreasing their count? } \\
\text { - Are the groups 'closing the gap'? } \\
\text { - Further assess change within your groups }\end{array}$ \\
\hline$\downarrow$ \\
\hline $\begin{array}{l}\text { 5. Test the significance of change for individual small areas } \\
\text { - Does the evidence point to significant change for individual } \\
\text { small areas? } \\
\text { - Locate the areas of significant change } \\
\text { - Explore patterns of significant change }\end{array}$ \\
\hline
\end{tabular}

Note: This flow chart outlines the key steps involved in the analysis strategy. The flowchart assumes that the data being analysed is available at the Lower Layer Super Output Area level and is related to deprivation. For more detail on the issues and any of the techniques described see the full guidance document. The principles and steps outlined may be equally applicable at other levels and for other types of data but, as ever, care must be taken to ensure that your analysis is appropriate and valid. 
The flowchart provided in Figure 7.1 outlines the 5 stages of the strategy and the key steps within each stage. The following sections provide an introduction to each of these steps and the main issues to consider in carrying out the analysis.

\section{Stage 1: Selecting the data}

Before any analysis can be carried out it is obviously important to focus on a topic and find reliable and representative data for your analysis. No matter how obvious or inevitable these decisions may seem it is always worth checking your choice of data against the principles outlined in the following steps. Choosing the wrong data will almost always produce incorrect results - and potentially poor policy decisions.

\section{Select a topic of interest}

In most cases the topic will be obvious and will be decided by a particular problem or policy area - perhaps change in worklessness, crime or health conditions.

Before settling on the topic, however, it is important to consider what data are likely to be available. You will need reliable data at a suitable geographic level and in a comparable form for (at least) two dates.

If data are not available for your exact topic of interest it may be necessary to find a 'proxy' - that is, a strongly related variable from which to infer information about change. As noted above, for our case study we will use benefit claimant data as a proxy for 'worklessness'.

\section{Select data at a suitable geographic level}

Key points to consider when selecting an appropriate geography for your data are:

- You will usually want to use data at the lowest level available. For example if you are interested in neighbourhood level analysis look for data for Lower Layer Super Output Areas (LSOAs) or similar

- It is best to compare areas that are similar in terms of size. Usually this means population size, but may also refer to the number of dwellings, the working population or even, in some cases, the physical size of the area

- Wherever possible look for data on the same geography for both dates. It may be appropriate to compare between data for two different geographies as long as they are included in the same hierarchy (eg Super Output Areas (SOAs) and LA districts) but the resulting analysis will be at the higher level (in this example LA districts) and there will clearly be some extra work in combining values from the smaller geography

- Be aware that boundary changes during the study period will make analysis more difficult. Choosing a stable geography such as the Super Output Areas will minimise such problems

\section{Select a timeframe}

Again your topic of interest and the availability of data will strongly influence your choice of dates to compare between.

When considering data from different years it is important to ensure that the data collection processes are comparable. Have record collection or processing methods changed during the period? Have data definitions changed? Have laws, rules or eligibility criteria for requesting benefits changed?

In the case of data reported monthly or quarterly, you need to check whether the data demonstrate seasonal variation - that is, whether they vary on a regular annual cycle. If seasonality is present in the data, then comparisons should only be made on the same time period, for example comparing January in one year with January the next, rather than trying to compare January with August.

\section{Decide whether to use count or rate data (or both)}

The decision to use count or rate data in the analysis is based upon two considerations:

1) the purpose of the analysis, and

2) data availability and quality

\section{1) Purpose}

\section{- Rates}

Rates are most useful when the topic of concern relates to incidence or prevalence or when the topic has a strong relationship with population size. Examples might be the extent to which a disease occurs within a population or the proportion of the population achieving certain educational standards.

For example, when considering educational achievement for females, analysis of achievement rates will take account of the fact that there may be different numbers of females taking the exams each year. Counts of those passing exams would not reflect this - so demographic changes might influence the results as much as any real changes in exam results.

\section{- Counts}

Counts might be used where there is less relationship between the population size and the count (for example, the number of serious road traffic accidents in an area) or where the focus is more clearly on resources or stock (for example, the number of house available for rent).

You might consider using a scatter plot to investigate the strength of relationship between the variable of interest and the population in each area and so inform your decision here.

\section{2) Data availability and quality}

In order to use rates, you need an accurate measure of the 'population at risk'. For example, to calculate the incidence of measles in infant schools in an area, you need to know the number of children in such schools. Such information 
may not be available, or the information may be considered too inaccurate to be relied upon. If you do wish to use rates you need to be sure of the quality of both the variable of interest and the population denominator. Unreliable denominators will always result in unreliable results.

Case Study

\section{Selecting the data}

For our study we have selected 'worklessness' as the topic of interest.

As there is no single source of data collected on 'worklessness' at a low geographic level, we have decided to use data taken from the Department for Work and Pension's Working Age Client Group (WACG) dataset for 2004 and 2005 which is available at LSOA level. The benefit claimant counts for various benefit count variables were combined to form what we describe as the 'out-of-work benefit claimant count', and we accepted the limitations of using the data as a proxy for worklessness.

Where we are carrying out analysis that compares results between local authorities (Stage 2) we will use rates because population differences between the individual LAs has a strong bearing on the comparison. For the rest of the analysis, where the number of people workless is more relevant for policy makers, we will use counts.

Summary of data chosen for the case study:

- Topic of interest: Worklessness

- Data: Out-of-work benefit claimant count data as a proxy for 'worklessness'

- Geography: data aggregated to LSOAs within a fictitious local authority

- Timeframe: 2004 to 2005

- Counts or rates: Rates (for context) and Counts (for change analysis)

\section{Stage 2: Understand the context of the analysis}

Understanding the wider context of the analysis and conditions in neighbouring areas will often provide a better understanding of what is going on in your area and how significant local changes really are. For example, if the rest of the region is experiencing an economic downturn, then even a small decrease in the worklessness rate in a local authority area might be considered encouraging.

In some cases a consideration of context will not be appropriate and you may wish to jump direct to small area analysis (Stage 3) but normally the following steps will be useful.

How does your local authority compare with its region?

The first step is to consider where your LA falls compared with other areas in the region. A simple place to start is to produce a table of counts or rates for the region and district for both time periods. You can then consider any differences between regional and LA results. When carrying out region or LA level analyses it is normally best to use rates, as variations in population are likely to influence the results.

Case Study

Table 7.2 Out-of-work benefit rates for our case study LA compared with its region

\begin{tabular}{llrr}
\hline & & & $\begin{array}{r}\text { Government } \\
\text { Office Region }\end{array}$ \\
\hline 2004 Count & 58,540 & 661,180 \\
& Working age population & 295,240 & $4,918,640$ \\
& Rate (\%) & 19.8 & 13.4 \\
\hline \multirow{2}{*}{2005 Count } & 58,430 & 663,940 \\
Working age population & 296,510 & $4,982,070$ \\
& Rate (\%) & 19.7 & 13.3 \\
\hline & Change in rate 2004 to 2005 & -0.1 & -0.1 \\
\hline
\end{tabular}

The out-of-work benefit rates at the local authority and GOR level are both showing a very small decrease of 0.1 per cent over this time period.

Not only is this change very small but it is also important to remember that the quality of rates depend a great deal upon the precision of the denominators used - in this case mid-year population estimates. Although the result here suggests that there has been very little change overall both at LA and GOR level, these findings need to be treated with some care.

We will consider the significance of changes at these levels, and also for individual LSOAs, later. 
How does your local authority compare with other local authorities?

Considering how your LA compares to its neighbours will often be useful. Accordingly, the next step in the strategy recommends that box plots are used to compare the spread of data for each LA. A box plot is a statistical graphic that provides a visual overview of a data distribution by showing the median, quartiles and spread for each area of interest. ${ }^{2}$

\section{Case Study}

The box plot in Figure 7.3 shows the spread of worklessness rates for local authorities by Government Office Region 2005, ordered by the median value. The other regions of England have also been included here because they provide further context and because the place of individual regions may be of interest.

The single box plot for London (the location of our case study data) shows how our area falls compared with the other LAs in the region. The solid green dot on the box plot locates our local authority's rate. Our LA's rate value is higher than the third quartile for the region (its value lies in the top 25 per cent of LAs in London). This shows that the rate of worklessness is quite high in comparison to the majority of other local authorities in the region.

\section{Figure 7.3 Box plot for each region, out-of-work benefit rate}

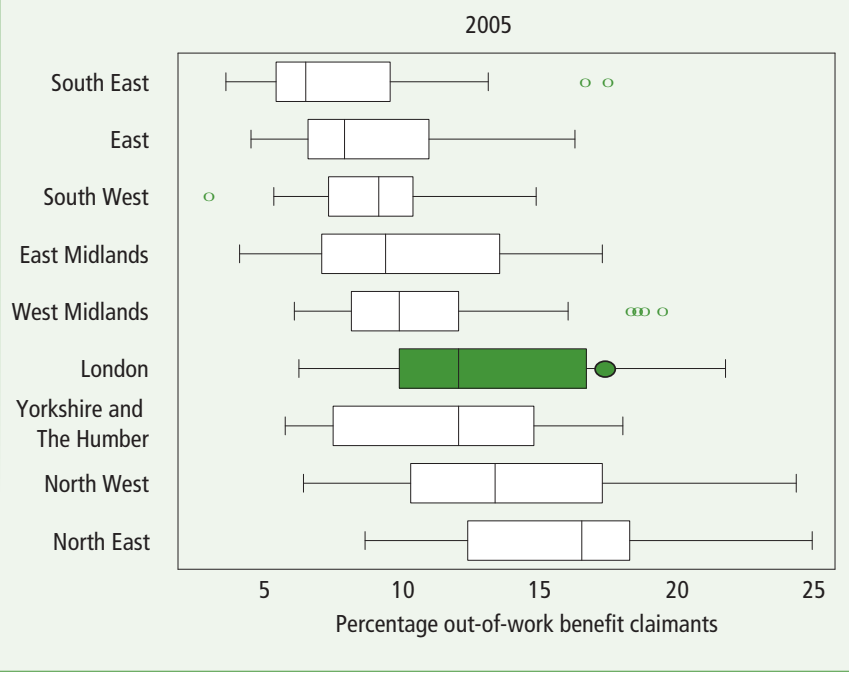

2 If you are unfamiliar with the use of box plots, see the full guidance document referred to at the start of this article or Regional Trends 40 (Page 18) for advice.
How do changes in your local authority compare with those in other local authorities in the region?

You can now examine more specifically the change between the two dates for which you have data - referred to here as time 1 and time 2 .

Table 7.2 showed one way to look at change - by subtracting the rates for time 1 from time 2 to calculate the difference. You can again use box plots to show the spread of the difference of rates over the same time period.

Case Study

Figure 7.4 shows a box plot of the differences in the outof-work benefit rates for the period 2004 to 2005 for LAs within each region. Data points below zero indicate a decrease in out-of-work benefit rate, while data points above zero indicate an increase. As noted earlier, these rates are created using estimates of working age population - and the changes in rates are all relatively small. Accordingly these results are, at best, only indicative and individual results need to be treated with caution.

\section{Figure 7.4 Percentage change in out-of-} work benefit claimants

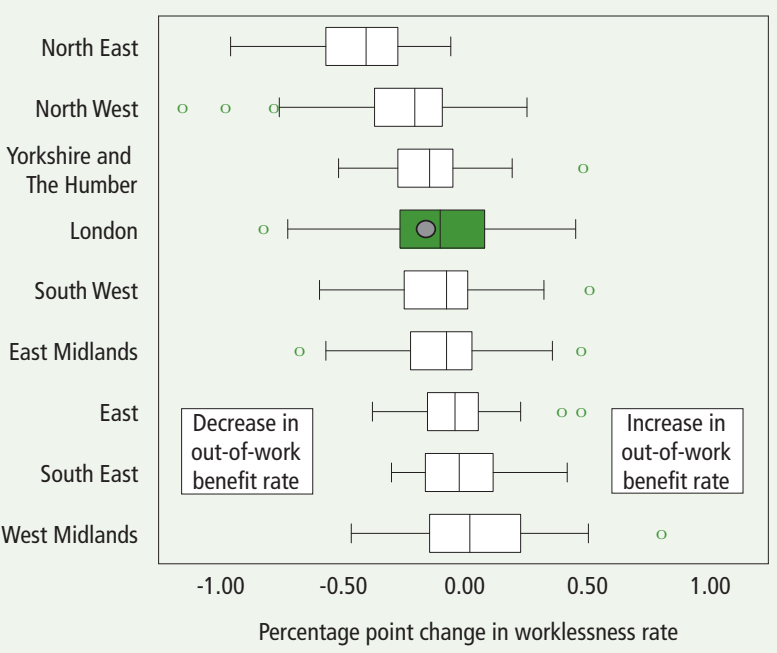

Our example local authority's difference value is again marked on the box plot. Although, as we found out before, our LA has a relatively high level of worklessness (as reflected in the benefit rates) it can be seen here that it has experienced a decrease in worklessness over the time period. It also appears to be 'improving' faster than just over half of the local authorities within the London region.

Again the results for all regions are shown for further context and interest. The region at the top, North East, has seen the biggest reduction in its rates from 2004 to 2005 , with the majority of the local authorities in the North East showing a small percentage point decrease. 
How are the data distributed within your local authority area?

Having looked at change over time for the local authority as a whole the next step is to explore the variability of small areas (for example, LSOAs) within the local authority.

The strategy recommends that you produce frequency histograms in order to study the distribution of values within the data.

Since the main policy interest in this type of analysis is likely to focus on reducing the number of claimants, the rest of our case study makes use of count rather than rate data.

\section{Case Study}

Figure 7.5 provides example histograms for each of our two years of interest - and the patterns are very similar. In both cases most LSOAs are towards the lower end of the distribution but there are many at the higher end too, with some even having benefit counts in excess of 400 . Nonetheless there are some subtle differences between the two years which we will investigate in later parts of the analysis.

\section{Figure 7.5 Histograms showing out-of- work benefit count in LSOAs for the example LA}
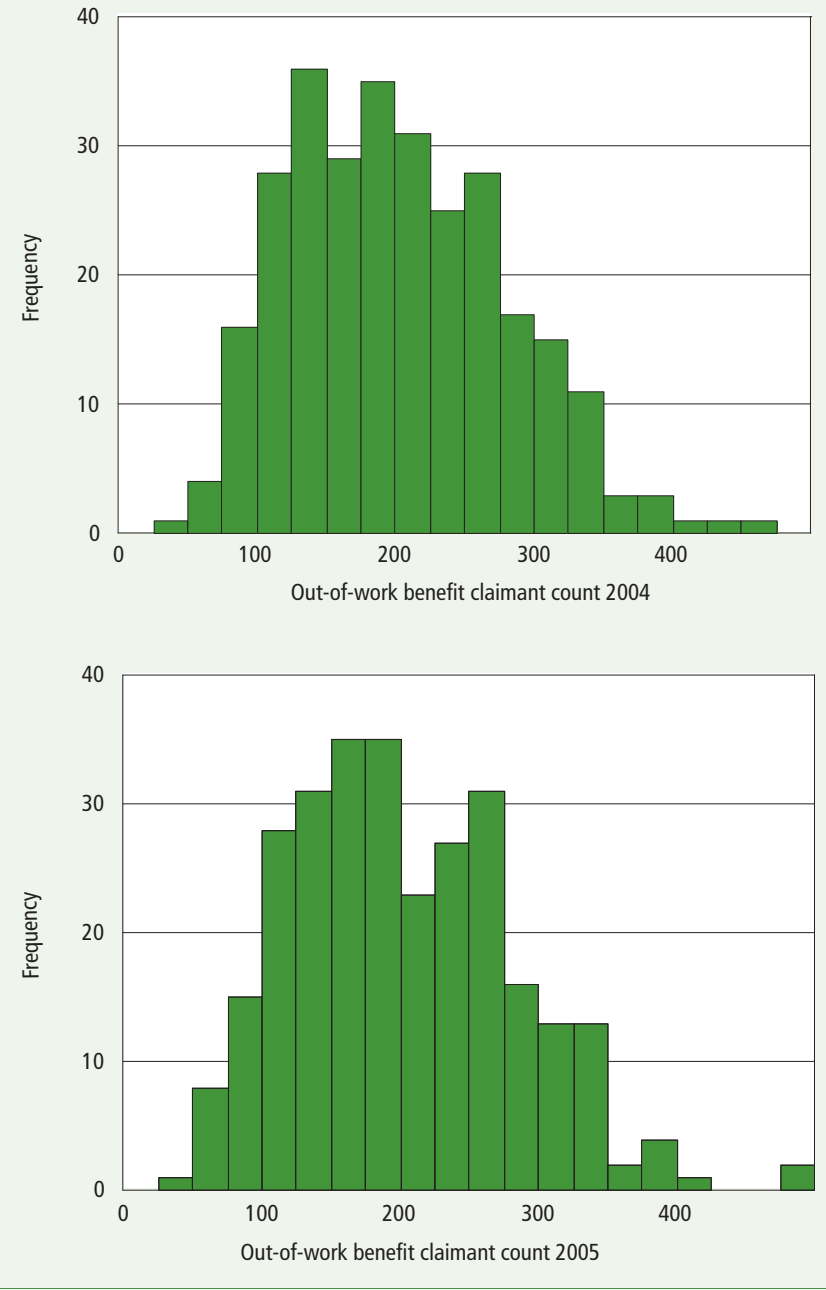

Has the overall spread of data changed in your area?

A number of measures for determining the spread of your data are possible and although discussion of these is outside the scope of this article, the strategy recommends using a statistic called the Median Absolute Deviation - described in detail in the full guidance document. Again you should produce statistics for both years in order to compare changes in the spread over the study period.

Are there any obvious spatial patterns within your local authority?

It is a good idea to look next at spatial patterns as they can reveal those areas contributing most to the change. This will reveal whether there are areas with high rates clustered together or if they are more evenly spread around the local authority.

The most obvious way to investigate spatial patterns is to map your source data in order to identify any patterns or 'hotspots'.

\section{Case Study}

Map 7.6 shows the distribution of benefits rates within the case study area and reveals some clear clusters of high counts - notably in the centre of the area.

\section{Map 7.6 Benefit counts in case study area by LSOA, 2004}

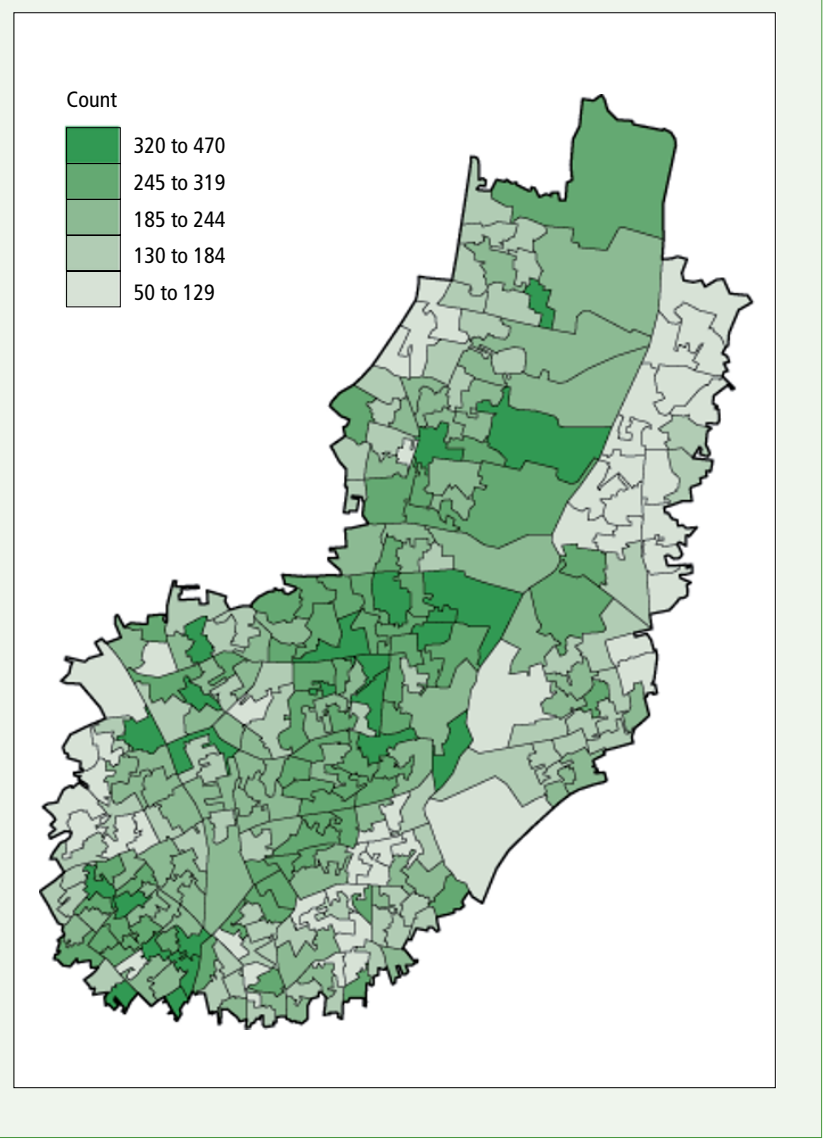


How has each LSOA in your local authority changed from time 1 to time 2?

The final stage in considering the context is to look at the change between time 1 and time 2 for each LSOA within the LA.

The following examples use a histogram, a scatter plot and a map to provide different perspectives on local change and specifically to identify which areas are contributing most to changes.

\section{Case Study}

\section{- Step 1: Histogram}

Figure 7.7 provides a histogram of differences in out-ofwork benefit counts in our study area - produced simply by subtracting the count for 2004 from that for 2005 .

Figure 7.7 Out-of-work benefit count difference, LSOA level, 2004 to 2005

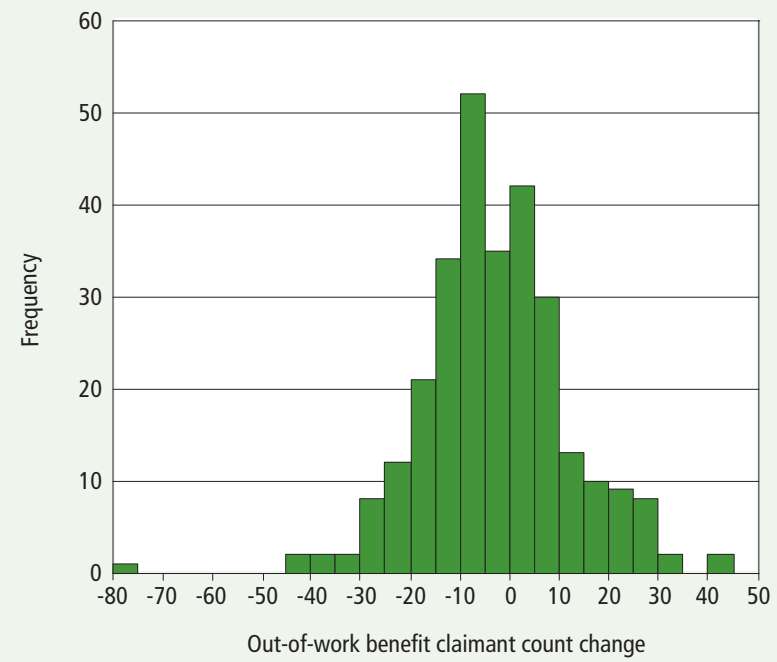

The differences for the LSOA's out-of-work benefit counts are evenly distributed about zero - a similar number of LSOAs have seen a decrease in out-of-work benefit counts as those that saw an increase. As well as a number of LSOAs with large increases in claimant count, we can see that one LSOA has decreased by around 80 claimants.

\section{- Step 2: Scatter plot}

Figure 7.8 shows the out-of-work benefit count for all the LSOAs in our local authority in 2004 and 2005 using a scatter plot. The 45-degree line on the graph represents equal claimant counts between the two time periods, that is, no change. Points above the line are LSOAs which have increased in count and those below have decreased. As suggested by the histogram, a similar number of LSOAs lie above and below the line. Interestingly the two LSOAs with the highest counts are seen to have experienced an increase in their claimant counts for 2005 .

\section{- Step 3: Map the change}

Finally, plotting the change on a thematic map allows us to identify spatial patterns.
Figure 7.8 Scatter plot, out-of-work benefit count, LSOA level 2004 and 2005

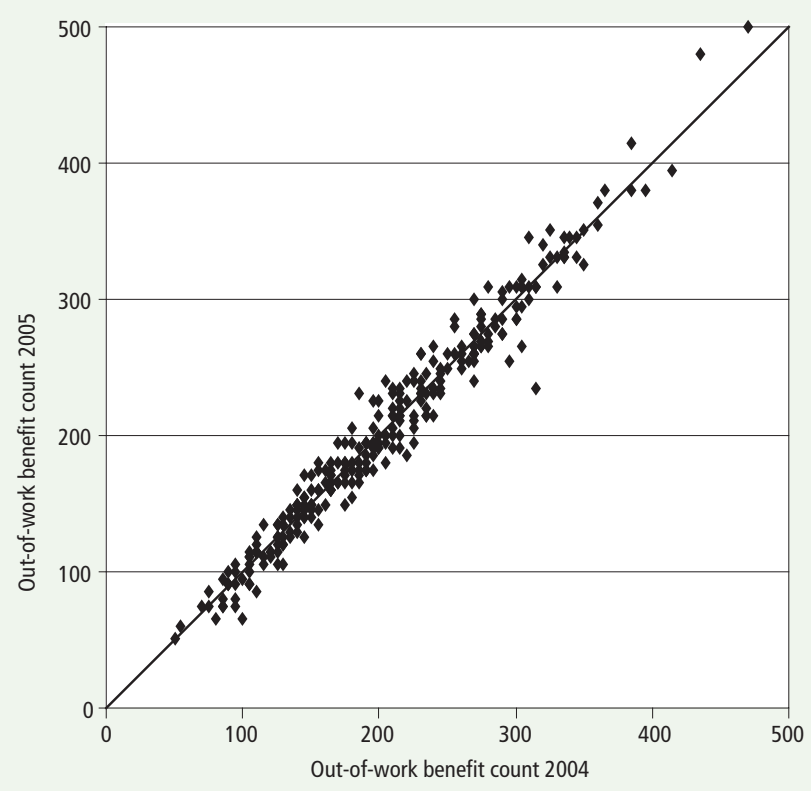

We can see from Map 7.9 that areas in our case study with increased claimant counts are largely in the central north of the LA but that elsewhere the pattern is very mixed. In many cases areas increasing in count lie immediately next to areas seeing reductions.

Later on we will consider techniques for testing how significant these local changes really are.

\section{Map 7.9 Change in out-of-work benefit by LSOA, 2004 to 2005}

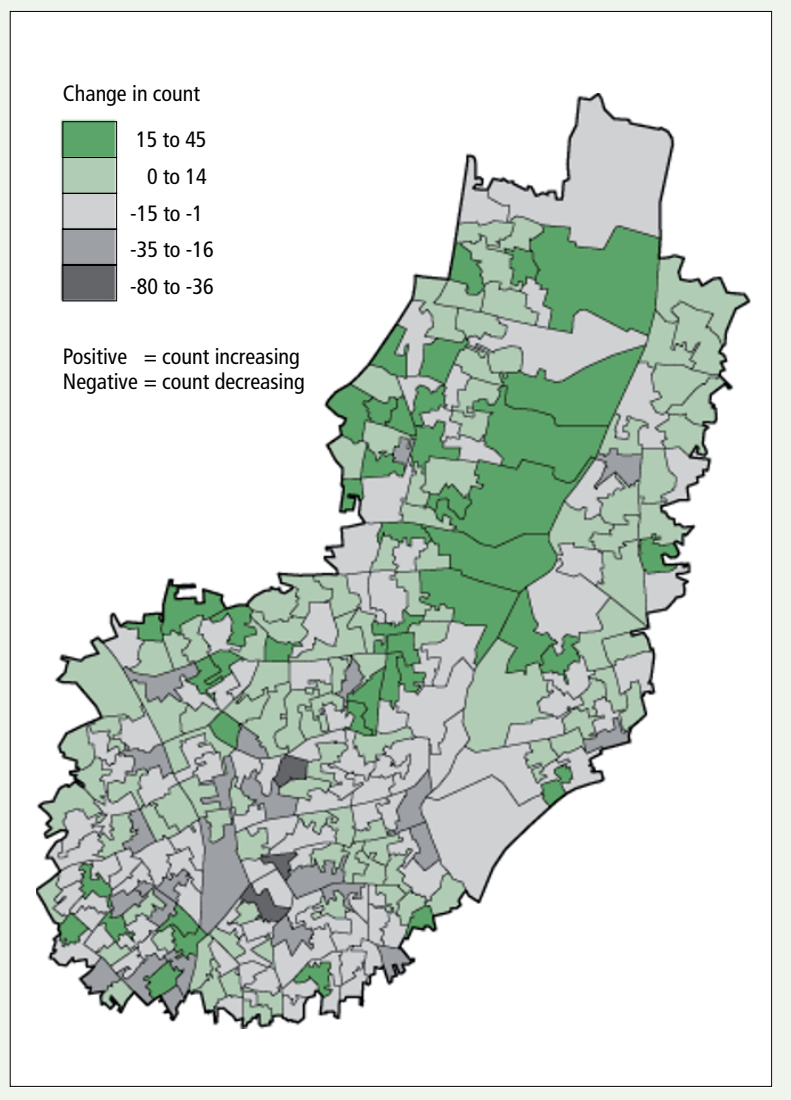




\section{Stage 3: Split your area into groups for analysis}

Having fully analysed the context of our data it is possible to move on to consider how separate groups within the data are changing.

In many cases when considering change over time the focus is on 'disadvantage' or a consideration of how the gap between well-off and less well-off areas has changed. Accordingly, the next stage will usually be to split the study area into separate groups for analysis. This will enable you to compare the two groups and to see if the gap between them is closing over time.

'Disadvantage' is a complex phenomenon which is difficult to define and quantify for analytical purposes. The full guidance document describes a set of statistically valid methods, ranging in terms of complexity, to help make decisions about choosing groups for analysis. It does not provide a prescription on how to allocate areas to groups but, rather, provides options and identifies the issues which need to be thought through.

In some cases the group of areas which you want to analyse will be defined by something other than disadvantage - say by poor performance on some criteria. Equally you may have a list of predefined areas - perhaps where there has already been some policy intervention. What is important is that analysts and policy makers use their knowledge of the area and topic concerned and fully consider the issues raised. The method chosen defines the group used for comparison and so can have a substantial influence on the results of analysis.

Even if you know the areas that you are interested in, it may be worthwhile trying other methods to validate your selection and to check if any additional areas ought to be included.

One key issue common to all approaches, however, is whether to use count or rate data in determining the split. The decision here will be entirely dependant upon the topic concerned, the nature of the data available and the purpose of the analysis. As discussed earlier, where the population concerned (that is, the denominator) varies greatly between areas or where the size of the population concerned is likely to have a direct influence on the count (for example, the influences of diseases) it may be appropriate to use rates. Equally for topics less closely related to the population of the area concerned (for example, crime, road accidents) counts may be more appropriate.

\section{Case Study}

For the purpose of our case study we have chosen a cutoff threshold of 245 - LSOAs with a count of 245 or higher are considered to be in our 'disadvantaged' group. When assessing the data we decided to add an additional LSOA to the group which had a high rate (43 per cent) but small count (100).

The cut off point of 245 is arbitrary in this case, chosen to demonstrate the next stages, but for real analysis the choice of these groups is fundamentally important. Different groups will produce different results - and poorly chosen groups can mask real differences and changes.

\section{Map 7.10 Location of disadvantaged areas in our example LA}

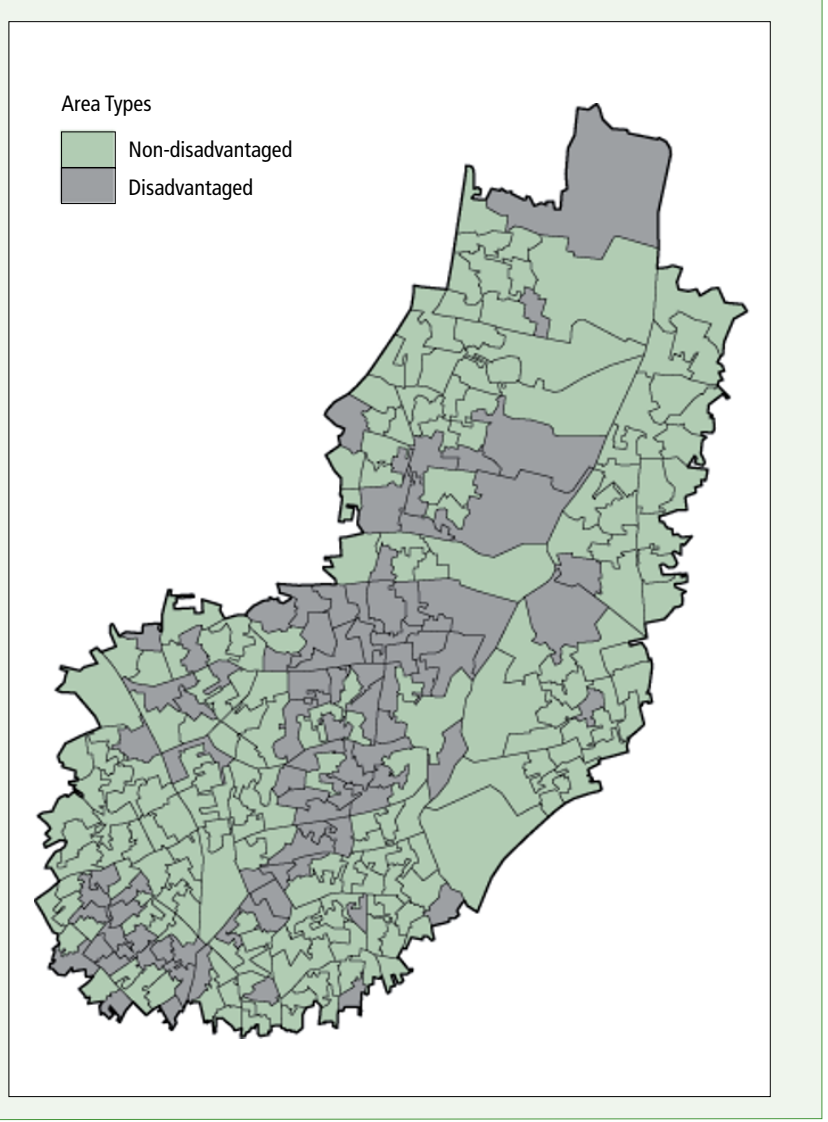

Nonetheless, this is a difficult area in which to set rules and you need to think carefully about the topic and available data before deciding upon your approach. Often it may be appropriate to run the method for both counts and rates, compare the differences and consider what these tell you about the area.

Once you have decided on a method for splitting and have created separate groups which properly represent the topic of interest, it is finally possible to move on to looking at how the areas have changed. 


\section{Stage 4: Understand how the groups are changing}

Splitting the local authority into groups enables you to compare the two groups and see if the 'gap' between them is reducing over time. Any closure in the gap may be the result of the disadvantaged group performing better - but could equally be the result of relative changes in both groups.

Again the flowchart in Figure 7.1 takes you through the main steps.

Has the spread of data in the upper-half of the data distribution changed?

Assuming that the main point of interest lies in the 'disadvantaged' areas, it is a good idea to analyse the 'upper half' of the data distribution - we refer to this as the right-hand spread. Although it does not specifically refer to the groupings created in Stage 3, it indicates whether the spread of the upper half of the data distribution in your area has increased or decreased, and this is where the more disadvantaged areas will be.

It is possible to look at the right hand spread by using percentiles (see Figure 7.11).

\section{Case Study}

\section{Figure 7.11 Percentiles of out-of-work benefit count, 2004}

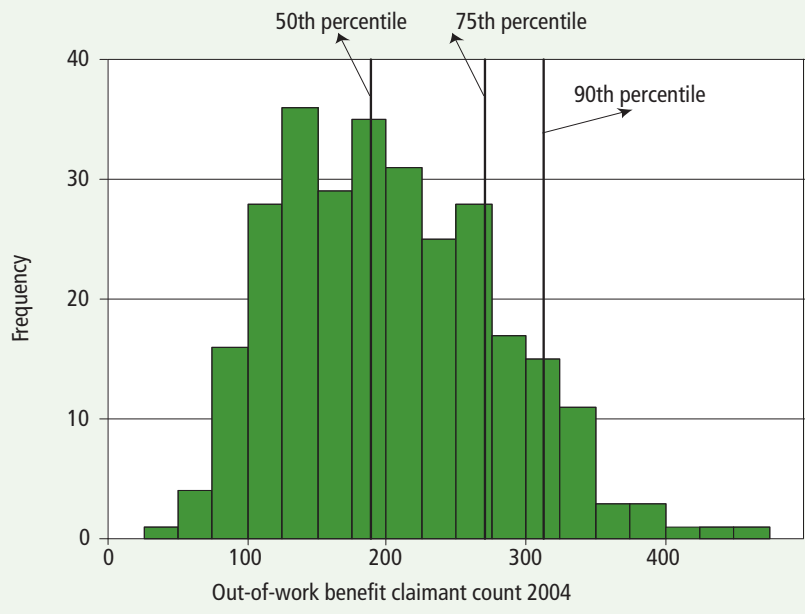

Taking the difference between the 90th percentile and the 50th percentile as a measure of spread, we can compare this measure of spread for two years to provide evidence of a reduction or increase in the right-hand spread.
Table 7.12 shows the percentiles of out-of-work benefit counts for 2004 and 2005 for our example LA. The largest percentile we have used is the 90th. This allows us to capture the spread over a wide range of areas whilst excluding those LSOAs where the data is likely to be most extreme (and whose inclusion might lead to incorrect conclusions).

\section{Table 7.12 Percentiles of out-of-work} benefit counts, 2004 and 2005

\begin{tabular}{|c|c|c|c|c|c|c|}
\hline & \multicolumn{2}{|c|}{ Percentile } & \multirow[b]{2}{*}{90} & \multirow[b]{2}{*}{ P90-P50 } & \multirow[b]{2}{*}{ P90-P75 } & \multirow[b]{2}{*}{ P75-P50 } \\
\hline & 50 & 75 & & & & \\
\hline 2004 & 195 & 270 & 310 & 115 & 40 & 75 \\
\hline 2005 & 195 & 260 & 310 & 115 & 50 & 65 \\
\hline
\end{tabular}

The table includes out-of-work benefit counts for the three percentiles, 50, 75, and 90 for each year. The righthand side of the table shows the differences in counts between the three percentiles. These differences represent the spread of data. For example, we see that the spread from the 90th to the 50th percentile remains constant at 115 counts (that is, the 90th percentile is 115 claimants higher then the 50th). We interpret this as no change in the right-hand spread.

Looking within the right-hand side of the claimant count distribution (the 75th percentile), we see that there has been a slight increase in the spread between the 90th and 75th percentile (from a count of 40 to 50) with a corresponding slight decrease in the spread between 75th and 50th percentiles (a reduction from 75 to 65). Although the right-hand spread has not increased, there is a slight change in the shape of the distribution of claimants with fewer LSOAs at the more extreme end.

The LA would seem to be improving slightly but we need to look more closely at what is happening within the groups. 
How are the groups increasing or decreasing their count?

This question moves us on from looking at the spread, to
A summary count of the changes within the group is the obvious starting point here.

\section{Case Study \\ Table 7.13 Number of LSOAs whose count have increased, decreased or not changed

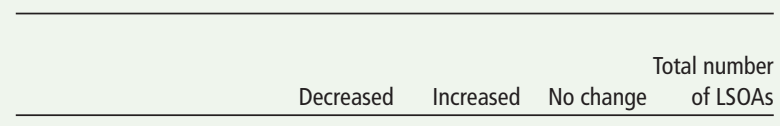

$\begin{array}{lrrrr}\text { Disadvantaged } & 46 & 33 & 9 & 88 \\ \text { Non-disadvantaged } & 88 & 83 & 26 & 197\end{array}$ \\ The disadvantaged group would seem to be doing rather better than the non-disadvantaged areas - with a higher proportion showing an improvement (decrease in the benefit count).}

These counts provide some information about the differences between the groups - but they are only a starting point. The next stage is to consider whether one particular group is performing better than the other, and whether the gap between the two groups has decreased.
Are the groups 'closing the gap"?

Our case study takes you through the four steps recommended in the strategy for identifying whether the groups are getting closer together.

\section{- Step 1: Calculate average change for each group}

\section{Case Study}

Table 7.14 summarises the change using individual count differences for each LSOA. The average change in each group is calculated by summing all of the differences and dividing by the number of LSOAs within the group.

\section{Table 7.14 Average change in out-of-work count}

Average change in counts (2004 to 2005)

\section{All LSOAs}

Disadvantage group Non-disadvantaged group

We can see that the disadvantaged group has reduced worklessness by 1.48 counts per LSOA compared with the non-disadvantaged group, which has reduced by 0.08 counts per LSOA. This shows that the nondisadvantaged group has seen virtually no change on average while the disadvantaged group has seen a small amount of change. This would suggest that the gap between the two groups has slightly reduced. Again we will consider later whether this change is large enough to be statistically significant. 


\section{- Step 2. Visualise this change using a box plot}

Next, the spread for each group can be visualised using a box plot - see Figure 7.15.

\section{Case Study}

The size of the boxes and the lines (or 'whiskers') that extend beyond them show the spread of the changes for each group. The spread of changes is very similar though some differences can be seen. The median for the disadvantaged group is slightly less than the median for the non-disadvantaged group, -5 and 0 respectively. Again it seems that disadvantaged areas have done marginally better than the rest. There are also some notable outliers for the disadvantaged group which indicate that there has been some considerable change for a few LSOAs in the LA - a decrease of 80 out-of-work benefit claimants for one LSOA, and an increase of 40 for two LSOAs, one in each group.

\section{Figure 7.15 Box plot of the out-of-work benefit changes 2004-05 for disadvantaged and non- disadvantaged groups}

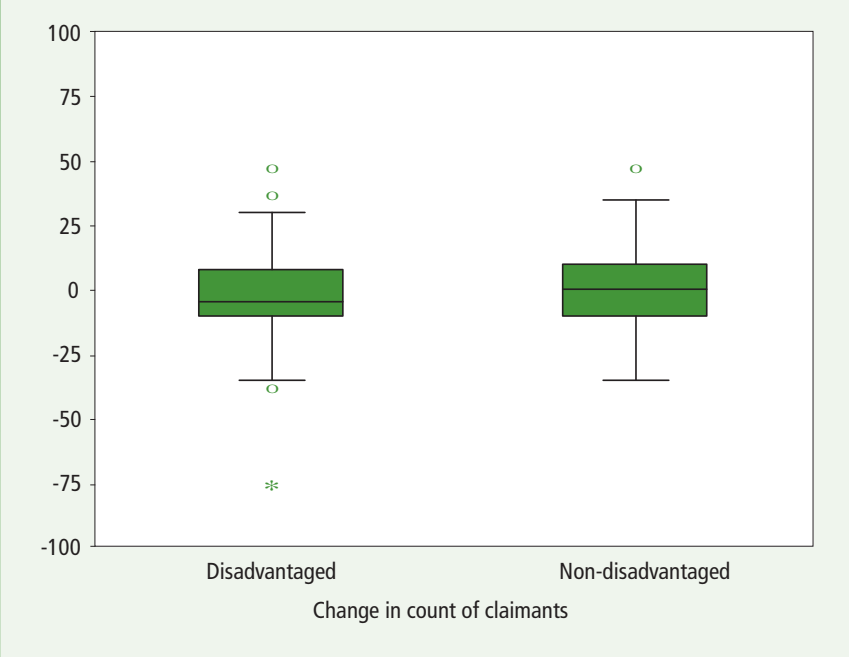

\section{- Step 3: Use a paired t-test}

A paired t-test allows us to determine whether the difference in change between the disadvantaged and non-disadvantaged groups is significant. See the full guidance document for details of how to apply this test.

\section{Case Study}

When running the paired t-test we find that the reported difference in average change in counts $(-1.48$ for the disadvantaged group, -0.08 for the non-disadvantaged group) is not statistically significant.

\section{What does this mean?}

First, it is confirmation that our intuition when analysing the box plot in Figure 7.15 - that there was minimal change between the two groups - was correct and that the two distributions are quite similar. More importantly, it tells us that we cannot say with confidence that the gap is closing between disadvantaged and nondisadvantaged groups for our local authority over the time period 2004 to 2005 . However attractive it may be to claim such an improvement, the evidence does not prove a statistically significant change.

\section{- Step 4: Further assessing changes within your groups}

Once you have assessed the differences between your groups, you may wish to explore further what is happening within the groups - for example: Is the change dominated by males or a particular age group? Does it vary with ethnicity? How are the extreme areas changing in comparison to the other disadvantaged areas? There is no prescribed way to carry out this analysis as it depends on what you want to find out, but our guide provides advice and some ideas for further analysis.

\section{Case Study}

Although our local authority is clearly changing, this section has highlighted how difficult it is to infer statistically robust change for groupings over a one or two year period. This is partly due to the characteristics of our example local authority, and partly due to the nature of change in worklessness for small areas.

Now we have seen that there are such changes, we can now think about whether individual LSOA changes are significant in terms of worklessness. 


\section{Stage 5: Test the significance of change for individual small areas}

This stage helps us to assess whether the change for each individual LSOA has been statistically significant. A significance test will show which areas have had change in excess of that which might be due to 'noise' in the data. Critically, areas of significant change should be investigated further to establish what has led to the change.

The significance testing can be computed using either rates or counts. For the purpose of the example in this guide we have, again, used count data.

Does the evidence point to significant change for individual LSOAs?

It is possible to test if significant change has occurred for each LSOA within the local authority and to consider the relationship to our 'disadvantaged' and 'non-disadvantaged' groups.

\section{- Step 1. Apply the Standardised Difference Method}

For every LSOA we can calculate the difference in counts over the two years (that is, 2005-2004).

\section{Case Study}

For our case study this shows us that 134 LSOAs had a negative change (fewer claimants), 116 LSOAs a positive change (more claimants) and 35 LSOAs no change at all. This does not, however, tell us if a statistically significant change has taken place.

To test how significant these changes are, we calculate a measure called the 'Standardised Difference' for each LSOA. This allows us to identify which LSOAs have changes bigger than you would expect from random variation.

The standardised difference is actually calculated by dividing the difference for each LSOA by the standard deviation ${ }^{3}$ (for all of the LSOAs). The full guidance document provides more details on the Standardised Difference calculation.

If the absolute Standardised Difference is more than 2 (that is, the change in an LSOA is greater than 2 standard deviations) then the LSOA is considered to have undergone a significant change.

\section{- Step 2. Decide on thresholds for the significance test}

Several thresholds can be used to test for different levels of likelihood. For example, when using a threshold of 2, for normally distributed data, you would expect that around 5 per cent of the LSOAs would lie outside of this value, split to both ends of the distribution (2.5 per cent in each end). You can, however, also use a threshold of 3 standard deviations to identify any areas with extreme change. It should be rare for LSOAs to fall beyond this extreme threshold and any that do are likely to warrant further investigation.

\section{Case Study}

Results for our case study LA are as follows:

\section{- Two standard deviations}

Using two standard deviations, 19 LSOAs displayed significant change. Of these LSOAs, 14 were within the disadvantaged group and five the non-disadvantaged. Of the disadvantaged areas nine had higher counts in 2005 in comparison with 2004. Of the non-disadvantaged, three had increased counts.

\section{- Three standard deviations}

We also assessed those LSOAs which had an absolute standard difference of above three and found that three LSOAs were identified. Two LSOAs had got worse by 45 counts and one LSOA had improved by 80 . These areas are definitely worth further investigation, ideally using local knowledge.

3 Standard Deviation is the most commonly used measure of the dispersion of data. For details on how to calculate the standard deviation see the guidance section of the Neighbourhood Statistics website at: www.neighbourhood. statistics.gov.uk 


\section{- Step 3. Locate the areas of significant change}

The areas of significant change can then be mapped.

Case Study

We created a map for our case study LA displaying the results of significance tests performed at two and three standard deviations.

\section{Map 7.16 Case study: Significance of changes 2004-2005 for disadvantaged areas}

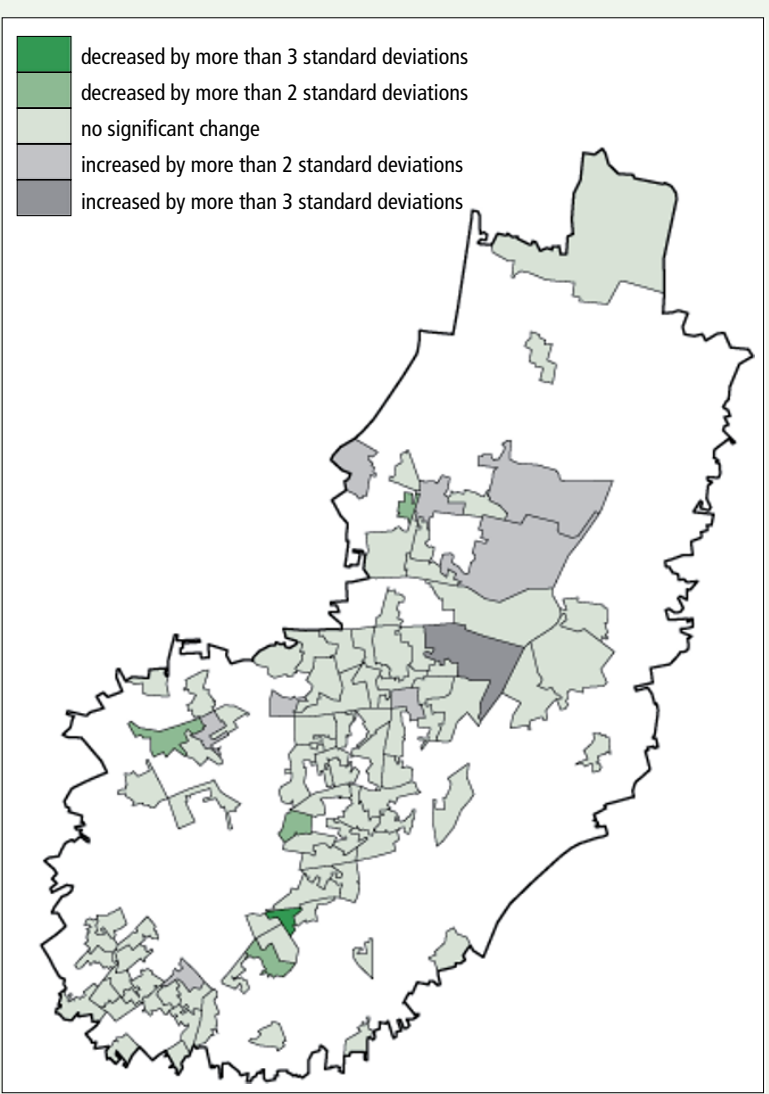

Map 7.16 shows the disadvantaged LSOAs deemed to have significantly changed and Map 7.17 the non-disadvantaged areas. We find that those disadvantaged LSOAs that have got worse are mainly in the centre of the local authority, and those which have improved are in the south.

\section{Map 7.17 Case study: Significance of changes 2004-2005 for non- disadvantaged areas}

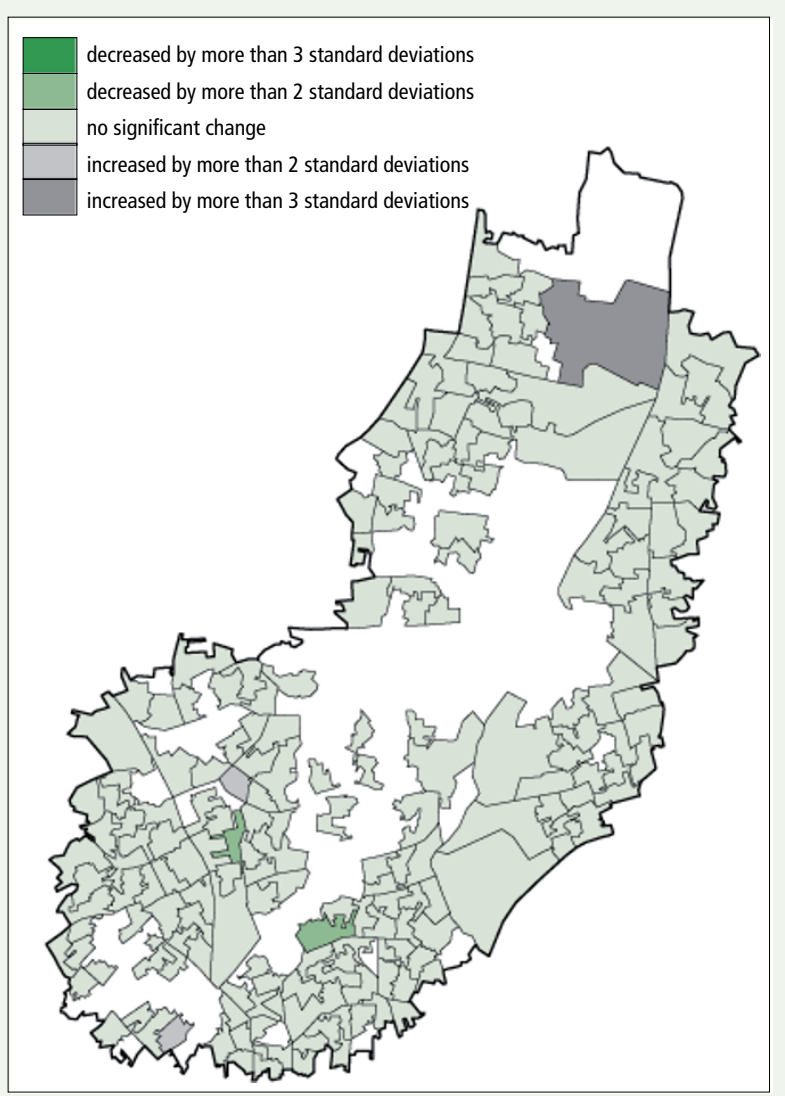




\section{Explore patterns of significant change}

The next step should be to use line plots to look at how individual LSOA have changed over the period.

The line plots help to visualise the size and direction of change for the significantly changed LSOAs and whether these LSOAs are clustered or spread evenly along the line. Critically they also show if any areas have changed from the non-disadvantaged to the disadvantaged group and vice versa.

Case Study

Figure 7.18 shows the out-of-work benefit count for all LSOAs in the LA for 2004 and 2005. The green line is drawn at a value of 245 - the 2004 threshold used when we split the groups. The data have been placed on separate lines for the disadvantaged (shown by black points) and nondisadvantaged (shown by green points) groups, to show the movements within and between these groups. Note that rounding of the data will mean that each point shown on the graph may represent several LSOAs (since LSOAs have the same out-of-work benefit count).

Those $19^{4}$ LSOAs identified as having a significant change are linked with lines to show the direction of the change (for example a left to right line shows an increase in benefit count). The line plot shows the magnitude of the individual significant changes and which group they belong to.
All of the LSOAs which had a significant change had an outof-work benefit count above 185, except one LSOA. For the highest values all of the significant changes were for LSOAs which saw an increase in their out-of-work benefit count.

It is interesting to look at the 2004 threshold (a count of 245 shown by the green line) to see how many LSOAs moved above or below it from 2004 to 2005 . Five LSOAs have moved from being classed disadvantaged to non-disadvantaged and six LSOAs have moved the other way. Of these 11 LSOAs, only four were found to have a significant change, showing again that the threshold level is arbitrary but important. Small changes in either LSOA values or in the threshold chosen will move individual LSOAs between groups.

4 Note the chart shows only 18 lines as two LSOAs have exactly the same rounded counts in 2004 and 2005. This line is shown slightly darker than the rest.

\section{Figure 7.18 Significant changes in out-of-work benefit data 2004-05}

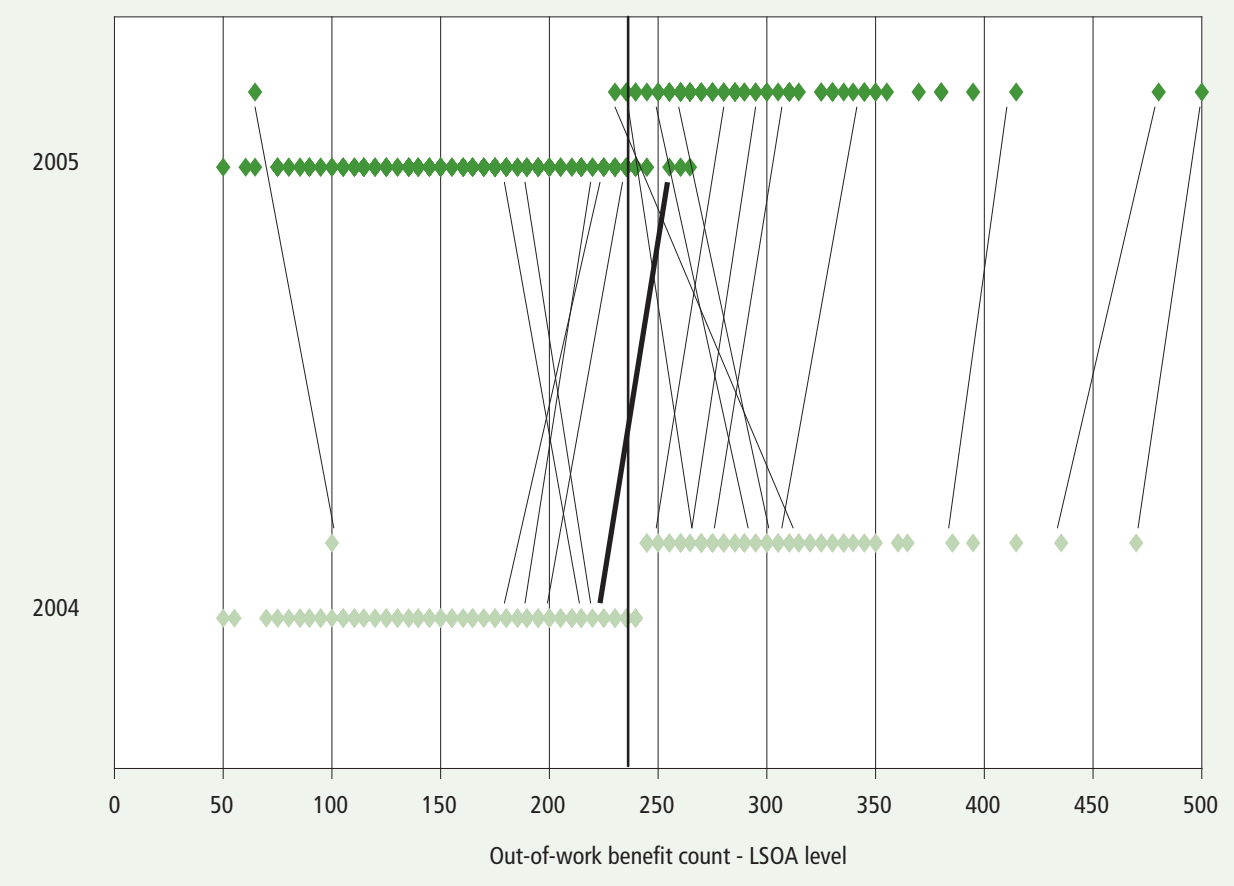




\section{Conclusions}

This article has aimed to show that although comparison of change over time has some complexities it is manageable when broken down into the stages outlined. Attention to the principles and issues summarised here - and particularly consideration of the statistical significance of any changes identified - should help in carrying out valid analysis.

If you want to carry out analysis for your own data, please study the more detailed guidance, which includes a more complete discussion of issues relating to data quality and variability.

We hope that the strategy and some of the techniques described here are useful locally and we strongly welcome any suggestions for future developments.

\section{Free Visualisation Tool}

A free interactive tool to illustrate the strategy and to support work on change over time was released early in 2009. The tool focuses on using benefits data as a proxy for 'worklessness' and automates the creation of box plots, maps and histograms for every LA in the country. If you would like a copy of the tool, please contact: Better.info@ons.gsi.gov.uk

\section{Office for National Statistics}

\section{Related titles from the Office for National Statistics}

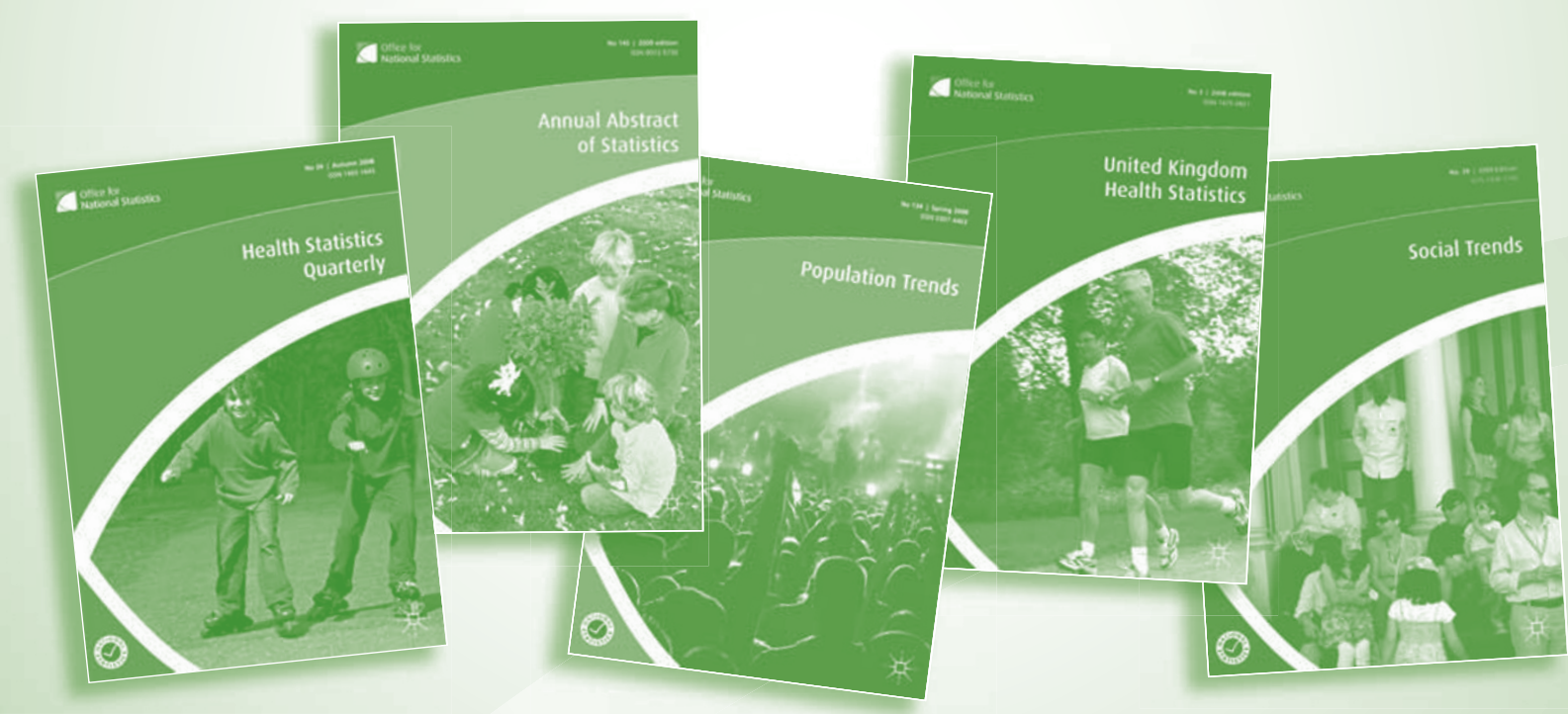

www.palgrave.com/ons/ 